\title{
Enhancement of aberrantly modified integrin-mediated cell motility in multicellular tumor spheroids
}

\author{
BYOUNGJAE KIM ${ }^{1,2}$, NU-RI IM ${ }^{1}$, TAESEOK DANIEL YANG ${ }^{3}$, JIAN KIM ${ }^{1}$, \\ KWANG-YOON JUNG ${ }^{1}$, TAE HOON KIM ${ }^{1}$ and SEUNG-KUK BAEK ${ }^{1}$ \\ ${ }^{1}$ Department of Otolaryngology-Head and Neck Surgery, College of Medicine; ${ }^{2}$ Neuroscience Research Institute, \\ College of Medicine; ${ }^{3}$ School of Biomedical Engineering, Korea University, Seoul 136-705, Republic of Korea
}

Received July 19, 2019; Accepted January 29, 2020

DOI: $10.3892 / \mathrm{ijo} .2020 .5016$

\begin{abstract}
Multicellular tumor spheroids (MTSs) of malignant cells can display cell-cell and cell-matrix interactions, different from monolayer cultures. The objective of the present study was to examine difference in intercellular and cell-matrix interaction between monolayered cultures and spheroid cultures. Expression levels of cell adhesion molecules (CAMs) and epithelial-mesenchymal transition (EMT) signaling molecules in monolayered cells and MTS cells were compared. The motility of single cells dispersed from each culture was evaluated using a live-cell imaging device. The effect of an E-cadherin neutralizing antibody, DECMA, was also compared between the two cultures. Among various CAMs, only E-cadherin was increased in MTSs. The motility of single cells dispersed from MTSs was higher than that from monolayered cells. Compared with monolayered cells, the molecular weight (MW) of $\beta 1$ integrin was decreased during MTS formation, particularly during the early stage. This notable reduction was maintained when DECMA was used to treat MTSs. Additionally, the expression levels of the EMT signaling molecules Snail and ILK increased more in MTSs than in monolayered cells. The blocking of E-cadherin elicited increased expression levels of EMT molecules and cellular motility only in MTSs. In conclusion, the alteration of E-cadherin expression and presence of low-MW $\beta 1$ integrin
\end{abstract}

Correspondence to: Professor Seung-Kuk Baek or Professor Tae Hoon Kim, Department of Otolaryngology-Head and Neck Surgery, College of Medicine, Korea University, Anam-dong 5-ga 126-1, Seongbuk-gu, Seoul 136-705, Republic of Korea

E-mail:mdskbaek@gmail.com

E-mail: doctorthk@gmail.com

Abbreviations: ATC, anaplastic thyroid carcinoma; CAM, cell adhesion molecule; ECM, extracellular matrix; EMT, epithelialmesenchymal transition; FAK, focal adhesion kinase; GAPDH, glyceraldehyde 3-phosphate dehydrogenase; ILK, integrin-linked kinase; MTS, multicellular tumor spheroid; ZO-1, zonula accludens-1

Keywords: spheroid, metastasis, motility, adhesion molecule, thyroid carcinoma in MTS may enhance cell motility via the upregulation of EMT signaling molecules that may be intensified by blocking E-cadherin.

\section{Introduction}

Multicellular tumor spheroids (MTSs) have been established as an in vitro model for the systematic study of tumor responses to therapy $(1,2)$. They are generally considered to be improved models when compared with two-dimensional cell cultures for predicting in vivo responses to drug treatment (3). The MTS system has been widely used as a model to study microenvironmental effects on basic biological mechanisms in cancer research, such as proliferation, differentiation, cell death, invasion and metastasis $(4,5)$.

As the three-dimensional organization of cancer cells in spheroids is different from the cellular organization of cancer cells in monolayer cultures, a previous study has suggested that the expression of cellular adhesion molecules (CAMs) known to be responsible for cell-cell and cell-matrix interactions is altered in MTSs compared with that in monolayer cultures (6). Given that alteration of CAM expression is associated with invasion and metastasis (7), biological behaviors of cells in MTSs may be different from those of cells grown in monolayer cultures, particularly cellular migration and invasion.

In the early phase of tumor progression, epithelialmesenchymal transition (EMT) is induced by loss of E-cadherin, a cell-cell adhesion molecule and epithelial marker, with disaggregation of cancer cells from one another (8). However, previous studies have revealed that loss of E-cadherin itself is not enough to induce EMT or mediate EMT signaling functions that may induce cancer cells to complete later steps of tumor progression more rapidly (9-12). In addition, E-cadherin-mediated cell-cell adhesion is regulated by downstream signaling of integrin-extracellular matrix (ECM) adhesion (13). This crosstalk between these two adhesions can modulate cellular motility (14). Thus, regulation of EMT by E-cadherin loss might be associated with the aberrated production of integrin-mediated adhesion, which is closely related to the EMT signaling molecules Snail or integrin-linked kinase (ILK) (15-17).

In the current study, it was hypothesized that altered expression of adhesion molecules in MTSs might affect the 
motility of spheroid-derived cells. To test this hypothesis, the expression patterns of CAMs, E-cadherin and $\beta 1$ integrin, and EMT signaling markers were investigated in anaplastic thyroid carcinoma (ATC) cells grown as monolayer cells or MTSs, and in E-cadherin blocked cells. In addition, cell motility was monitored using real-time phase-contrast imaging.

\section{Materials and methods}

Cell culture conditions. The ATC cell line, FRO, was generously gifted by Dr Young Suk Jo (Yonsei University, Seoul, Korea) and showed a negative mycoplasma result when tested using a Mycoplasma Detection kit (Lonza Group, Ltd.). For monolayer culture, cells were grown in RPMI-1640 medium (Welgene, Inc.) supplemented with L-glutamine, $100 \mathrm{U} / \mathrm{ml}$ penicillin-100 $\mu \mathrm{g} / \mathrm{ml}$ streptomycin and $10 \%$ heat-inactivated fetal bovine serum (Young In Frontier Co., Ltd.). When cells reached $80-90 \%$ confluency, they were trypsinized and subcultured to maintain the cell line. For experiments, $1 \times 10^{5}$ cells were cultured in six-well plates and harvested on the designated day: Monolayer day (MLD) 2-5.

For spheroid cultures, $1 \times 10^{4}$ cells/well in $200 \mu 1$ RPMI-1640 medium were plated onto an ultra-low-attachment-surface 96-well round-bottom plate (Corning Inc.) and centrifuged at $440 \mathrm{x}$ g for $3 \mathrm{~min}$ at room temperature (RT). These spheroids were incubated at $37^{\circ} \mathrm{C}$ with $5 \% \mathrm{CO}_{2}$ for up to 6 days: Spheroid day (SPD) 1-6. After plating, $100 \mu 1$ medium was replaced with $100 \mu \mathrm{l}$ fresh medium every other day. Spheroids were examined every day with a Motic AE31 inverted microscope. They were harvested on SPD 2, 4 and 6.

In order to block E-cadherin, treatment with an E-cadherin neutralizing antibody (DECMA, sc-59778, mouse monoclonal anti-human; $5 \mu \mathrm{g} / \mathrm{ml}$; Santa Cruz Biotechnology, Inc.) was conducted for $24 \mathrm{~h}$ at $37^{\circ} \mathrm{C}$ in monolayer and spheroid cultures, respectively.

Western blotting. For western blot analysis, monolayer cells or spheroids were extracted with $5 \mathrm{X}$ Laemmli sample buffer and $5 \% \beta$-mercaptoethanol by boiling for $10 \mathrm{~min}$. After determining the protein concentration using bicinchoninic acid assay, the same amount $(20 \mu \mathrm{g} / \mathrm{well})$ of the extracts were separated on 8 or $10 \%$ SDS-polyacrylamide gels (as specified for each antibody below) and transferred to nitrocellulose membranes. Non-specific binding sites on the membranes were blocked with skimmed milk for $90 \mathrm{~min}$ at RT. Membranes were then blotted with primary antibody against E-cadherin (rabbit polyclonal anti-human; sc-7870, 1:1,000, 8\%; Santa Cruz Biotechnology, Inc.), zonula accludens-1 (ZO-1; rabbit polyclonal anti-human; cat.no.61-7300, 1:1,000,8\%; Invitrogen; Thermo Fisher Scientific, Inc.), occludin (rabbit polyclonal anti-human; cat. no. 71-1500, 1:1,000, 10\%; Invitrogen; Thermo Fisher Scientific, Inc.), $\beta 1$ integrin (rabbit polyclonal anti-human; 4706S, 1:1,000, 8\%; Cell Signaling Technology, Inc.), ILK (rabbit polyclonal anti-human; PA5-27484; 1:1,000, 10\%, Invitrogen; Thermo Fisher Scientific, Inc.), Snail (rabbit polyclonal anti-human; 3879S, 1:1,000, 10\%; Cell Signaling Technology, Inc.), paxillin (mouse monoclonal anti-human; sc-365059, 1:1,000, 10\%; Santa Cruz Biotechnology, Inc.) or focal adhesion kinase (FAK; rabbit polyclonal anti-human; 3285S, 1:1,000, 8\%; Cell Signaling Technology, Inc.) at $4^{\circ} \mathrm{C}$ overnight followed by incubation with secondary antibody (peroxidase conjugated goat anti-mouse, 115-036-003, 1:5,000; peroxidase conjugated goat anti-rabbit, 711-036-152, 1:5,000; Jackson ImmunoResearch Laboratories Inc.) for $6 \mathrm{~h}$ at $4^{\circ} \mathrm{C}$. Blots were visualized using a chemiluminescence kit (Santa Cruz Biotechnology, Inc.). Chemiluminescence was captured with a ChemiDoc system (Bio-Rad Laboratories, Inc.). Mouse monoclonal antibody to glyceraldehyde 3-phosphate dehydrogenase (GAPDH; mouse monoclonal anti-human; sc-47724, 1:2,000, 10\%; Santa Cruz Biotechnology, Inc.) was used to detect GAPDH, which served as a loading control. The density of each band was analyzed using ImageJ software (version 1.51i; National Institutes of Health).

Reverse transcription-quantitative polymerase chain reaction. Monolayer cells or spheroids were resuspended with QIAzol lysis reagent (Qiagen, Inc.) to extract total RNAs by serial treatments with chloroform (Sigma-Aldrich; Merck KGaA), isopropanol (Duksan Pure Chemicals Co., Ltd.) and $75 \%$ ethanol in diethyl pyrocarbonate water. mRNAs were then transcribed into cDNAs using a cDNA Synthesis Master mix (amfiRivert cDNA synthesis Platinum Master mix; GenDEPOT) with the following temperature protocol: $5 \mathrm{~min}$ at $25^{\circ} \mathrm{C}, 50 \mathrm{~min}$ at $42^{\circ} \mathrm{C}$ and $15 \mathrm{~min}$ at $70^{\circ} \mathrm{C}$ ).

The prepared cDNA was amplified and quantified with a real time thermal cycler system (TP800/TP860; Takara Bio, Inc.) using SYBR Green Master mix (Qiagen, Inc.) with the following primers: E-cadherin, forward: 5'-TGCTCTTGC TGTTTCTTCGG-3' and reverse: 5'-TGCCCCATTCGTTCA AGTAG-3'; $\beta 1$ integrin, forward: 5'-TTCGATGCCATCATG CAAGTTG-3' and reverse: 5'-CCATCTCCAGCAAAGTGA AACC-3'; and GAPDH, forward: 5'-GAGTCAACGGAT TTGGTCGT-3' and reverse: 5'-TTGATTTTGGAGGGA TCTCG-3'. The thermal cycling conditions were $15 \mathrm{sec}$ at $95^{\circ} \mathrm{C}$ and $45 \mathrm{sec}$ at $60^{\circ} \mathrm{C}$. Data were analyzed using the $2^{-\Delta \Delta \mathrm{Cq}}$ method (18).

Immunocytochemistry. Spheroids were harvested from 96-well plates on days SPD1, 3 and 6, and then fixed with $4 \%$ paraformaldehyde overnight at RT. Fixed spheroids were then treated with ethanol and isopropanol and embedded in paraffin blocks for tissue sectioning. After deparaffinization and rehydration in descending grades of ethyl alcohol, 5- $\mu \mathrm{m}$ sections were quenched with $0.3 \%$ hydrogen peroxide in methanol for $30 \mathrm{~min}$ at RT. Non-specific sites were blocked with $3 \%$ normal goat serum (Vector Laboratories, Inc.) for $30 \mathrm{~min}$ at RT. Slides were incubated with primary antibody to E-cadherin (sc-7870, 1:50; Santa Cruz Biotechnology) overnight at $4^{\circ} \mathrm{C}$. The next day, slides were treated with goat biotinylated anti-rabbit IgG $(\mathrm{H}+\mathrm{L})$ secondary antibody (PK4001, 1:200; Vector Laboratories Inc.) for $1 \mathrm{~h}$ at RT. Antigen-antibody complexes were detected using an avidin-biotin complex detection system (Vectastain ABC kit; Vector Laboratories, Inc.). Slides were stained with DAB substrate kit (Vector Laboratories, Inc.), rinsed in water, briefly counterstained with hematoxylin at RT for $10 \mathrm{sec}$, and washed in water. After mounting, slides were examined under an Olympus BX51 light microscope (magnification, $\mathrm{x} 100)$. Pictures were captured and controlled using Olympus DP72 and DP2-BSW software (version 2.2). 
Live cell time-lapse imaging and data processing. To capture live cell time-lapse images to assess cell motility, single cells dispersed from FRO spheroids on SPD2 and from monolayers of FRO cells on MLD3 were used. Monolayer cells treated with DECMA $(5 \mu \mathrm{g} / \mathrm{ml})$ were also monitored. A culture dish containing these cells was placed in a temperature $\left(37^{\circ}\right)$ - and $\mathrm{CO}_{2}(5 \%)$-regulated live-cell chamber mounted onto the stage of an inverted microscope (IX71; Olympus) with an objective lens (x20; numerical aperture 0.40). Time-lapse live cell images of single cells were captured at 1-min intervals for 2 days using a cooled CCD camera (ProgRes ${ }^{\circledR}$ MFcool; Jenoptic) with a spatial resolution of $\sim 0.5 \mathrm{~mm} /$ pixel. To trace the trajectory of a crawling single cell, acquired images were analyzed using ImageJ software (version 1.51i). The centroid of the cell body was calculated for each frame. The sequence of centroid positions $(\mathrm{x}, \mathrm{y})$ was converted into speed and displacement. Data from 10 cells are expressed as mean \pm SD.

Blocking of E-cadherin and spheroid spreading assay. As mentioned above, DECMA $(5 \mu \mathrm{g} / \mathrm{ml})$ was used to block E-cadherin for $24 \mathrm{~h}$ in spheroid culture. To determine the effect of DECMA on the motility of MTSs, spheroids at day 2 were transferred to an adhesive plate and the spreading ability was monitored for 2 days. The area of the spheroid was measured using Image J software (version 1.51i).

Statistical analysis. All statistical analyses were carried out using SPSS for Windows (version 16.0.0; SPSS, Inc.). Data in graphs are expressed as the mean \pm SD of at least three independent determinations. Differences between two samples were determined using Mann-Whitney U-test. Differences with $\mathrm{P}<0.05$ were considered significant.

\section{Results}

Morphological examination and expression of CAMs during spheroid formation by ATC cells. To understand the effect of the confluency of monolayer cells on the formation of spheroids, MTSs were formed from monolayer cells with different confluency. The MTSs were packed tightly at SPD2 and sustained up to SPD6. When an MTS was formed using MLD3 cells, which had been cultured for 3 days after seeding single cells to culture plates, it maintained a relatively uniform and round morphology. However, MTSs formed from monolayer cells with higher confluency (MLD4 and MLD5) exhibited a rough surface in the early stage of MTS formation, and the outer cells were loosened easily (Fig. 1).

Expression levels of CAMs in monolayer cells increased when cells were grown to confluency (Fig. 2A; MLD2-5). The expression of E-cadherin was observed to increase in the early spheroid-forming period (Fig. 2A and B; SPD2 and SPD4) but decrease during late spheroid formation (Fig. 2A and B; SPD6) regardless of monolayer cell confluency, indicating that a certain amount of E-cadherin was required to form a spheroid. Notably, the distribution of E-cadherin was dynamic as the spheroid formed. It was distributed more centrally in the early period but was distributed throughout the spheroid in the late period (Fig. 2C). However, the expression levels of ZO-1 and occludin decreased sharply when spheroid formation began (Fig. 2A).
Motility of dispersed cells from ATC spheroids is higher than that of dispersed cells from cell monolayers. To evaluate changes in cellular motility after MTS formation, cells dispersed from monolayer cells and spheroids on SPD2 were cultured as single cells and monitored by time-lapse image capture for 2 days. Microscopic morphology analysis showed that dispersed cells from MTSs were longer and thinner than those from monolayer cells (Fig. 3A; Videos S1-S4). The speed and displacement of the dispersed cells from MTSs increased significantly during the first day (D1) compared to those of monolayer cells. However, on the second day (D2), the speed and displacement of the dispersed cells from MTSs were not markedly different from those on D1; nor was there any difference between dispersed cells from MTSs and monolayer single cells on D2 (Fig. 3B and C).

Expression of EMT molecules in MTS formation is different from that in monolayer cells. When the expression of EMT molecules was evaluated during spheroid formation, the transcriptional expression of E-cadherin increased while the protein level of E-cadherin markedly decreased at SPD0 but then increased to a value comparable with that in the monolayer at SPD2. Regarding the expression of $\beta 1$ integrin, a complicated aspect was observed during spheroid formation. Although the expression of $\beta 1$ integrin increased when spheroid formation began, its molecular weight (MW) critically decreased. As spheroid formation proceeded, the expression of $\beta 1$ integrin decreased and the reduction in the MW of $\beta 1$ integrin was sustained. Interestingly, the expression levels of ILK and Snail increased with spheroid formation, which was not associated with the expression level of $\beta 1$ integrin but did appear to be associated with the MW of $\beta 1$ integrin (Fig. 3D and E). By contrast, in monolayer FRO cell culture, the expression levels of the CAMs E-cadherin and $\beta 1$ integrin increased, whereas those of the EMT molecules ILK and Snail decreased with increasing confluency (Fig. 3F).

Altered expression of CAMs and EMT molecules by blocking E-cadherin in MTSs enhances cell motility. When the E-cadherin neutralizing antibody DECMA was used to treat monolayers or MTSs derived from FRO cells, the expression levels of E-cadherin and $\beta 1$ integrin decreased in both culture conditions. In addition, the EMT markers paxillin and FAK were expressed at lower levels in DECMA-treated cells. However, the expression levels of EMT signaling molecules ILK and Snail decreased in monolayer cells but increased in MTSs (Fig. 4A). To validate the different reactions to E-cadherin neutralizing antibody between monolayer cells and MTSs, motility assays were performed. E-cadherin-blocked monolayer cells moved more slowly than non-treated monolayer cells, whereas DECMA-treated MTS cells migrated further than non-treated MTS cells (Fig. 4B-D).

\section{Discussion}

In the present study, MTS cells expressed low-MW $\beta 1$ integrin and upregulated EMT signaling molecules, including ILK and Snail, which increasing the motility of the cells. By contrast, highly confluent cell monolayers exhibited low expression levels of ILK and Snail but high expression levels of CAMs; 
A

Monolayer culture

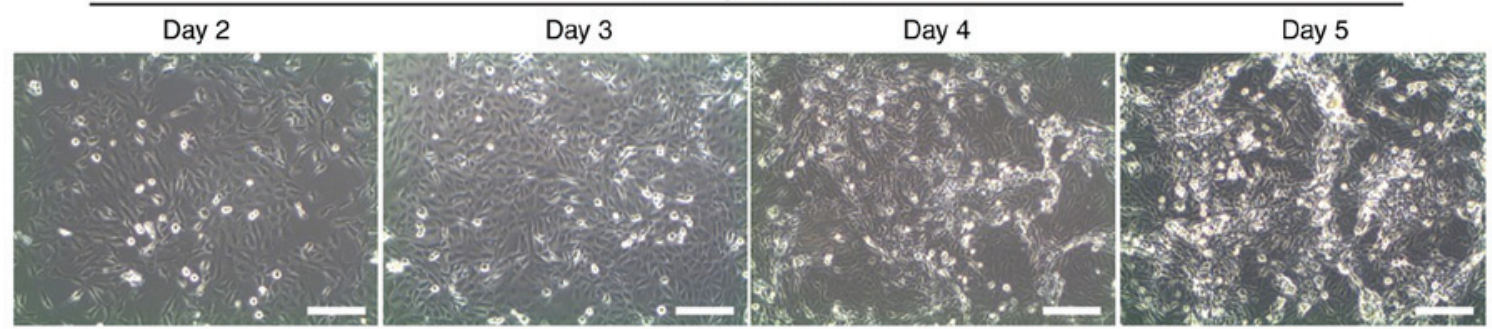

B

Spheroid culture

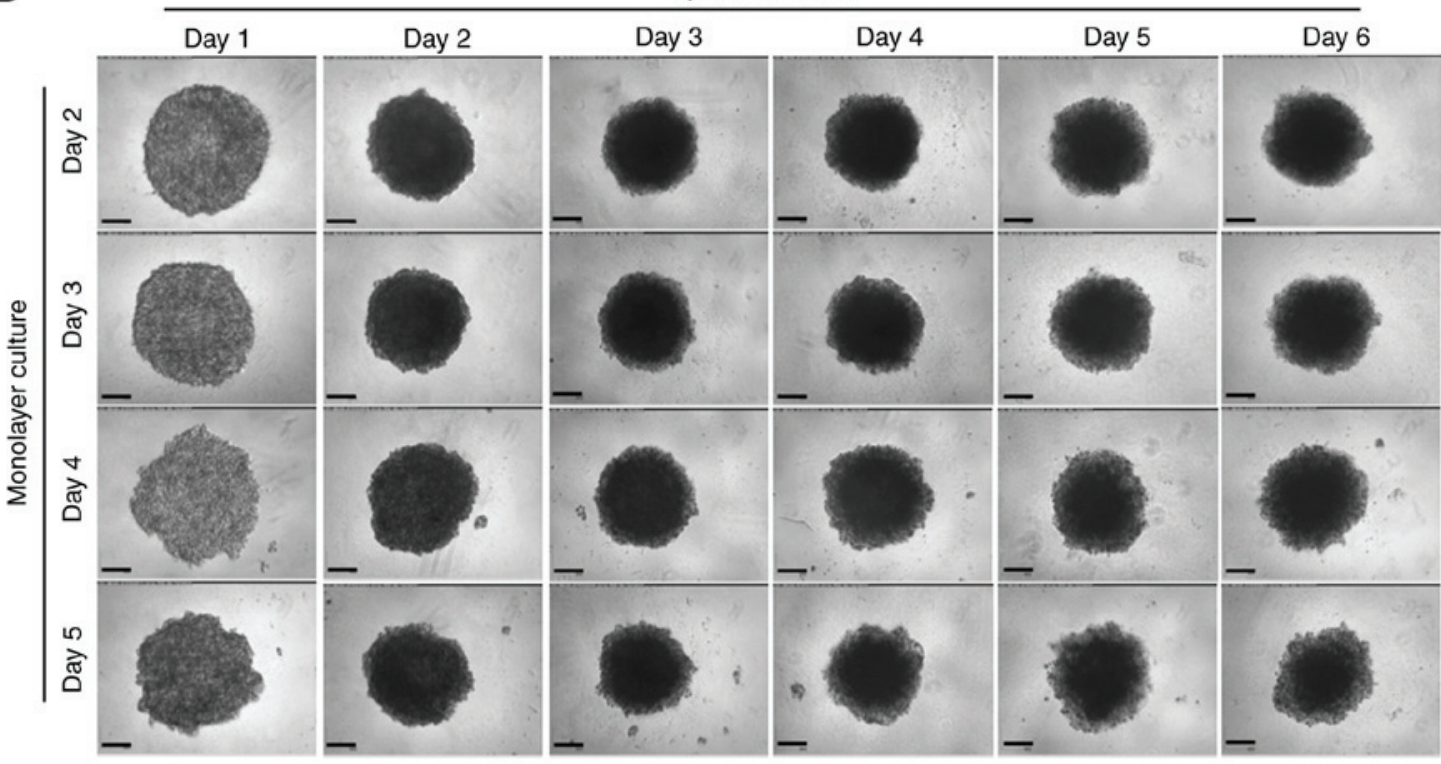

Figure 1. Morphological inspection of monolayer cells and MTSs. (A) FRO cells were cultured in plates for monolayer growth. Cells reached full confluency at day 3 and were overgrown from day 4. (B) MTSs grown from dispersed cells obtained by trypsinizing day 2 to day 5 monolayer cells with different confluency. Scale bar, $100 \mu \mathrm{m}$. MTS, multicellular tumor spheroid.

A $\mathrm{ML}$ $\mathrm{ML}$

SP

$\mathrm{ML}$

$\mathrm{SP}$

$\mathrm{ML}$

$\mathrm{SP}$

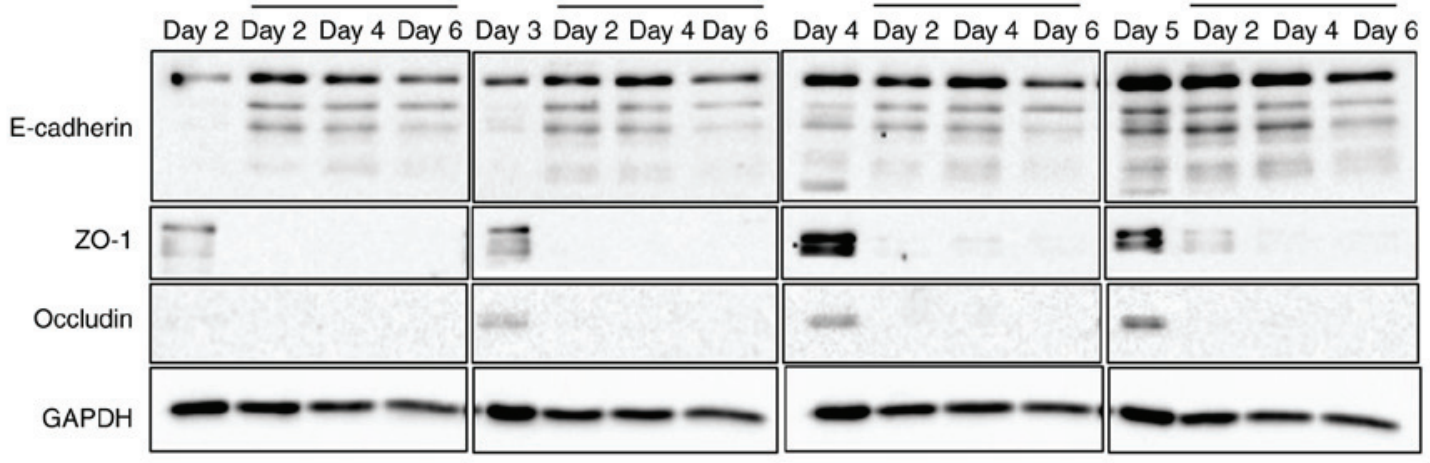

B
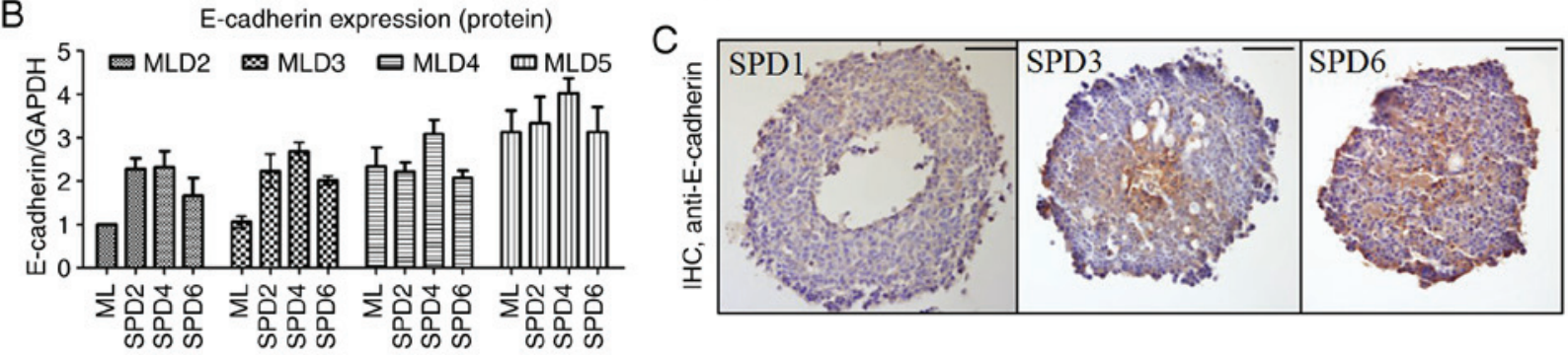

Figure 2. Expression of cell adhesion molecules in MTS formation. (A and B) Expression levels of the cell-cell adhesion molecules E-cadherin and ZO-1, and occludin were examined by western blotting. (A) The spheroids were made from day 2 to day 5 monolayered cells. The expression of E-cadherin was sustained until day 6 in MTSs, unlike that of the other proteins tested. (B) Quantification of the western blotting results. (C) Immunocytochemistry revealed that E-cadherin was expressed at the center of the MTS on day 3, and then evenly across the entire MTS on day 6. ML, monolayer; SP, spheroid; D, day; MLD, monolayer day; SPD, spheroid day; MTS, multicellular tumor spheroid; ZO-1, zonula accludens-1. Scale bar, $100 \mu \mathrm{m}$. 

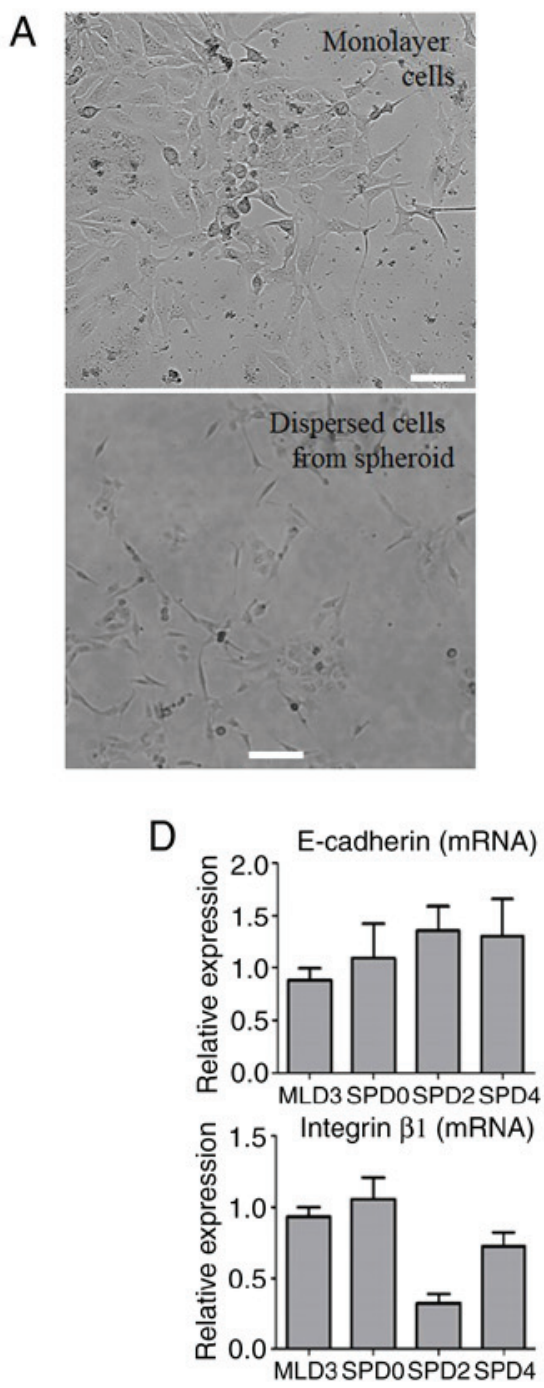

B
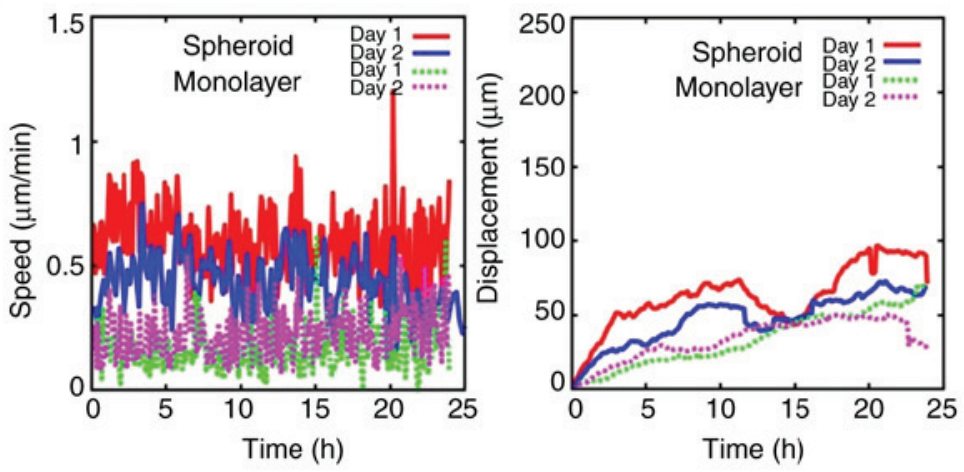

C

\begin{tabular}{lcclcc}
\hline & \multicolumn{2}{c}{ Speed $(\mu \mathrm{m} / \mathrm{min})$} & & \multicolumn{2}{c}{ Displacement $(\mu \mathrm{m})$} \\
\cline { 2 - 3 } \cline { 5 - 6 } & $\begin{array}{l}\text { Dispersed cell } \\
\text { from spheroid }\end{array}$ & $\begin{array}{c}\text { Monolayer } \\
\text { single cell }\end{array}$ & & $\begin{array}{c}\text { Dispersed cell } \\
\text { from spheroid }\end{array}$ & $\begin{array}{c}\text { Monolayer } \\
\text { single cell }\end{array}$ \\
\hline Day 1 & $0.61 \pm 0.59$ & $0.17 \pm 0.27^{\mathrm{a}}$ & & $60.15 \pm 38.36$ & $29.64 \pm 15.61^{\mathrm{a}}$ \\
Day 2 & $0.38 \pm 0.41$ & $0.22 \pm 0.35$ & & $39.78 \pm 22.18$ & $39.87 \pm 27.26$ \\
\hline
\end{tabular}

\section{E}

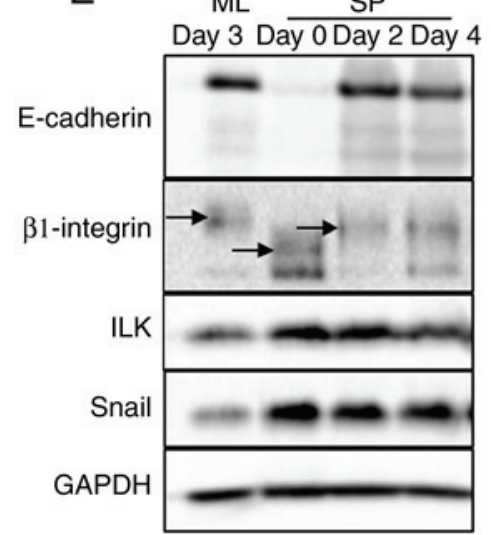

ILK (SP-protein)
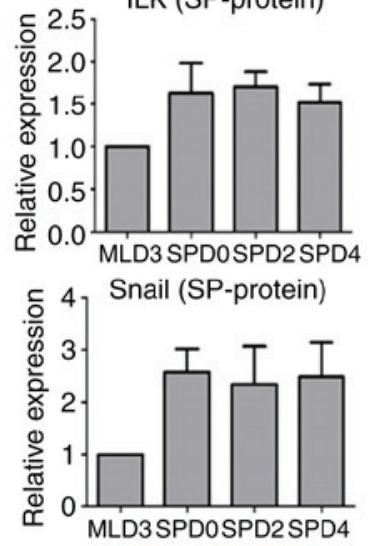

$\mathrm{F}$

ML

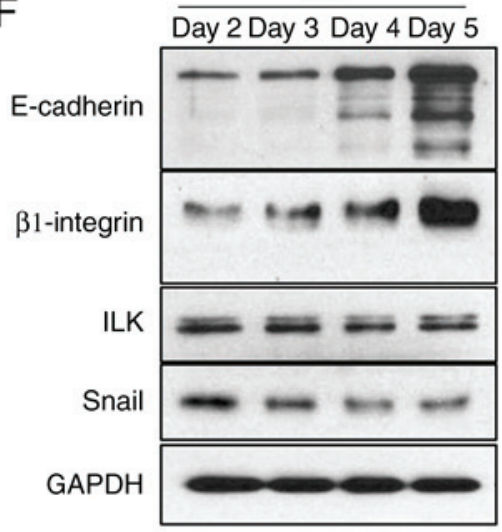

ILK (ML-protein)
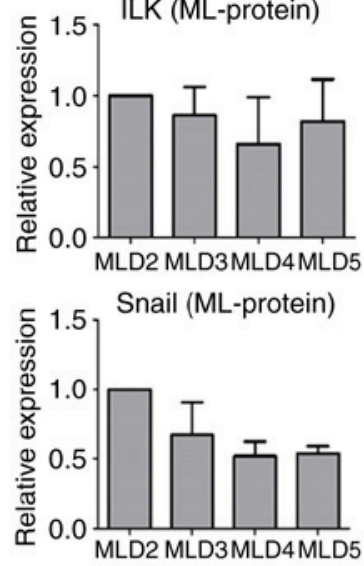

Figure 3. Increased motility of MTS cells with increased E-cadherin and EMT signaling molecules. (A) Images of single cells dispersed from cell monolayers and MTSs and cultured for $48 \mathrm{~h}$. (B) The speed and displacement of the dispersed cells were monitored (D1, 0-24 h; D2, 24-48 h) and compared statistically by analyzing 10 cells from each group. (C) The speed and displacement of dispersed MTS cells were increased more than those of dispersed monolayer cells after 24 h. ${ }^{a} \mathrm{P}<0.05$ vs. dispersed cell from spheroid on the same day (Mann-Whitney U test). (D) Transcriptional expression of E-cadherin was increased in MTS cells but that of $\beta 1$ integrin was variable. (E) During MTS formation, the molecular weight of $\beta 1$ integrin changed and expression levels of EMT signaling molecules increased. (F) Protein levels of cell adhesion molecules increased in monolayered cells at day 2 to day 5 while those of EMT signaling molecules decreased. MTS, multicellular tumor spheroid; EMT, epithelial-mesenchymal transition; ML, monolayer; SP, spheroid; D, day; MLD, monolayer day; SPD, spheroid day. Scale bar, $50 \mu \mathrm{m}$.

these changes decreased cellular motility. In monolayer and MTS culture conditions, when E-cadherin was blocked with neutralizing antibody, the expression levels of E-cadherin and $\beta 1$ integrin decreased; however, those of ILK and Snail 

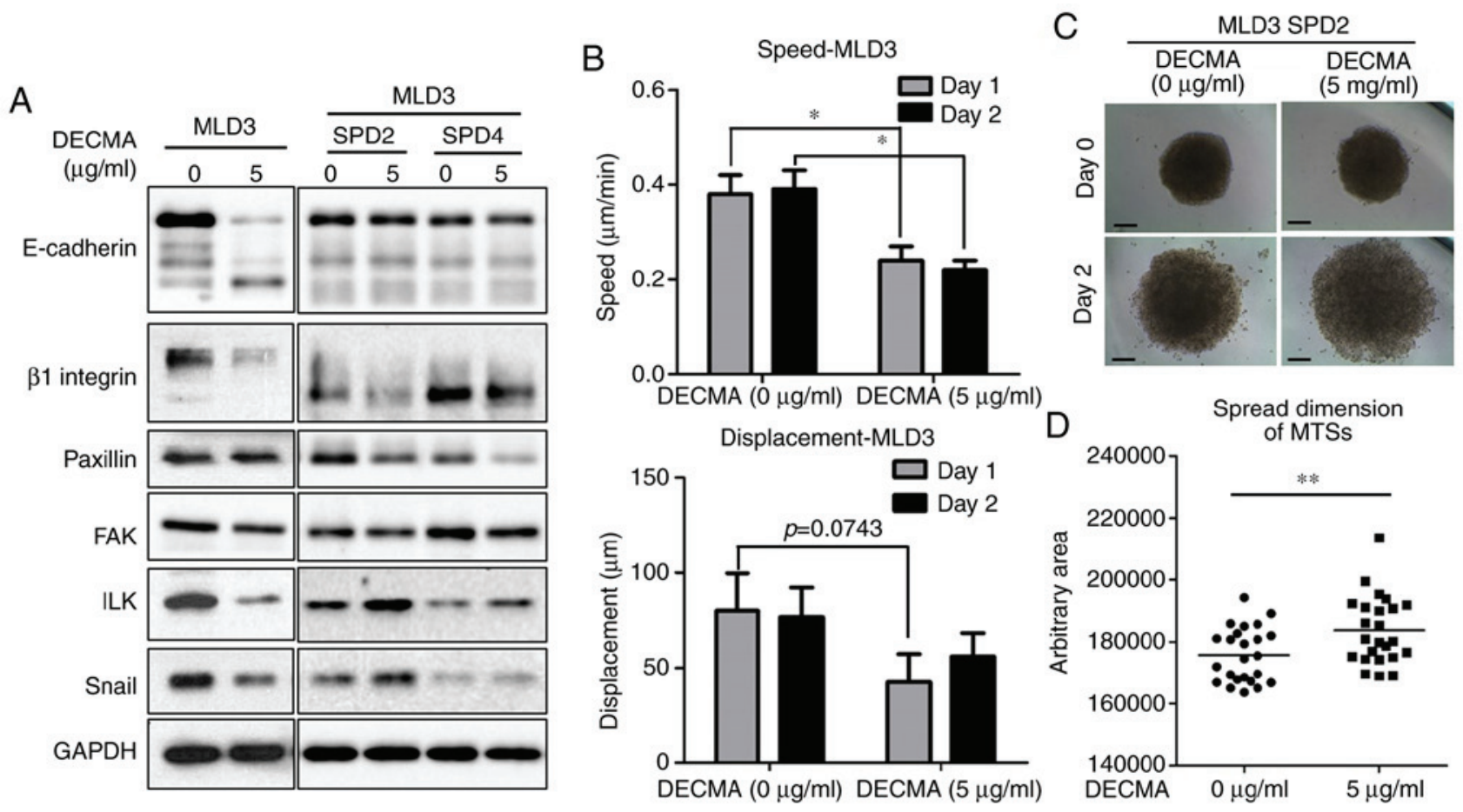

Figure 4. Low-MW $\beta 1$ integrin and EMT signaling molecules enhance cell motility. (A) Expression levels of cell adhesion molecules and EMT molecules after adding DECMA $(5 \mu \mathrm{g} / \mathrm{ml})$ to cell monolayers and MTSs for $24 \mathrm{~h}$ were examined. Only MTSs exhibited reduced MW $\beta 1$ integrin and DECMA-induced upregulation of ILK and Snail. (B) The speed and displacement of single cells dispersed from a cell monolayer were suppressed by DECMA. (C and D) The spreading extent of MTSs was increased by DECMA in a spreading assay. (C) Representative images and (D) quantified results. EMT, epithelial-mesenchymal transition; DECMA, E-cadherin neutralizing antibody; MTS, multicellular tumor spheroid; ILK, integrin-linked kinase; FAK, focal adhesion kinase, MLD, monolayer day; SPD, spheroid day. Scale bar, $100 \mu \mathrm{m}$. " $\mathrm{P}<0.05$ and ${ }^{* *} \mathrm{P}<0.01$ (Mann-Whitney U test).

differed according to the culture conditions: Upregulation of ILK and Snail and increased motility were observed in MTSs; downregulation of ILK and Snail and decreased motility were observed in monolayer cells (Fig. 5). These results demonstrate that when a two-dimensional cell condition was changed to a three-dimensional cell condition, in addition to simple structural changes, EMT signals were also upregulated by low-MW $\beta 1$ integrin.

ATC is one of the most lethal human neoplasms. Previous studies have reported a median survival time ranging from 3 to 10 months (19). Although ATC comprises $<2 \%$ of all thyroid carcinomas, it is responsible for $14-50 \%$ of annual deaths associated with thyroid cancer (20). The majority of patients with ATC present with advanced disease with regional and systemic metastases. ATC is the most undifferentiated and aggressive thyroid cancer. It is characterized by high metastatic spread (21). Among diverse differentiation levels of thyroid carcinomas, poorly differentiated ATC shows significantly lower expression of E-cadherin than other well- or moderately differentiated carcinomas $(22,23)$. Thus, an undifferentiated ATC cell line, FRO, expected to show high cell motility due to reduced levels of E-cadherin was used in the present study.

The expression of E-cadherin increased in proportion to the confluency of the monolayered cells and increased further when MTSs were formed. In addition to the expression level of E-cadherin, the cytological examination of MTSs suggested the importance of the differential distribution of E-cadherin in MTS formation. However, the motilities of cells dispersed from cell monolayers and MTSs were significantly different.
Cells from MTSs exhibited increased motility despite the high level of E-cadherin. In particular, MTS cells expressed low-MW $\beta 1$ integrin. Generally, when glycosylation of some proteins is changed, the function of these proteins in cancer cells may be affected, thus influencing several metastatic processes such as EMT, migration and invasion (24). A previous study has suggested that the glycosylation of integrins serves an important role in the metastatic process of cancer cells (25). In addition to glycosylation, several posttranslational modifications of integrin, including phosphorylation and palmitoylation, have been reported to regulate integrin activity in cancer cells $(26,27)$. Despite the diversity of the posttranslational modifications of integrin, glycosylation is important for numerous biological functions of $\beta 1$ integrin, including cell adhesion and migration $(28,29)$. Therefore, it is hypothesized that the enhancement of cancer cell motility with low-MW $\beta 1$ integrin observed in the present study may be associated with the aberrant glycosylation of $\beta 1$ integrin. However, this was not demonstrated empirically due to the complexity of the glycosylation mechanism and the diversity of the enzymes involved. Furthermore, glycosylation of integrins is regulated in thyroid cancer cells by several enzymes, including $\alpha 1-6$ fucosyltransferase and $\mathrm{N}$-acetylglucosaminyltransferase, that are less expressed in ATCs (30). Therefore, reduced MW $\beta 1$ integrin in FRO MTSs may represent a clinical state of undifferentiated carcinoma with increased metastatic ability.

Suppressive roles of E-cadherin in cancer progression have been well reviewed. The loss of E-cadherin is thought to enhance tumor invasiveness and increase the metastatic ability of many human carcinomas. In addition, as tumor 
A

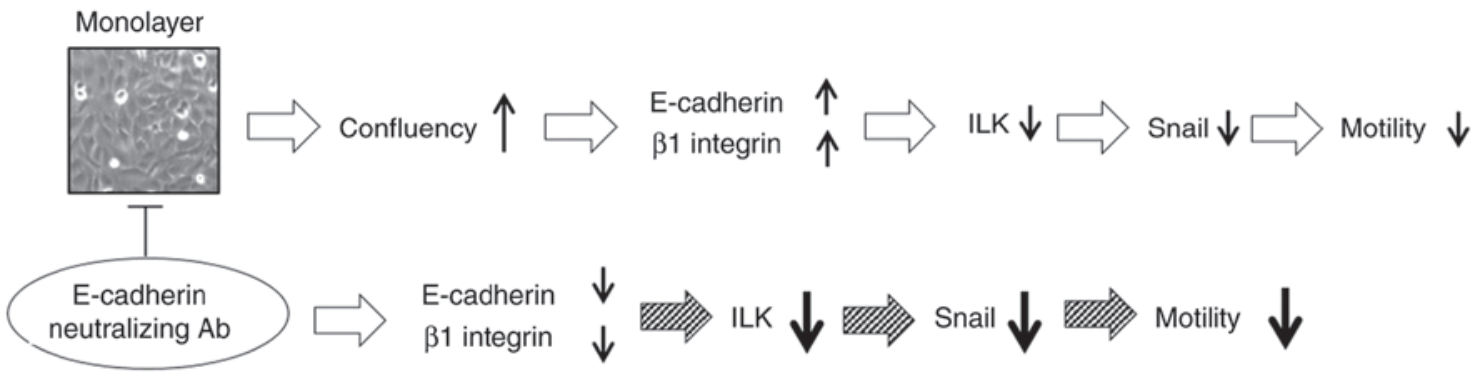

B

Spheroid
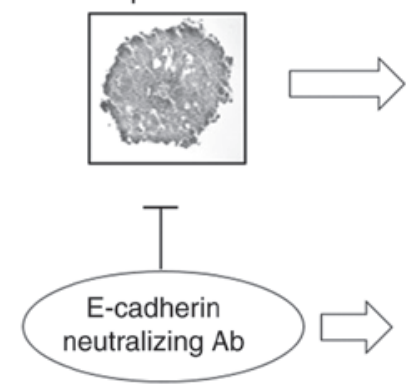

E-cadherin

Low-MW

$\beta 1$ integrin

E-cadherin

Low-MW

$\beta 1$ integrin

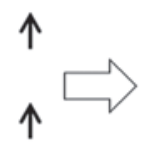

$\operatorname{ILK} \uparrow \square$ Snail $\uparrow$

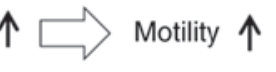

Figure 5. Differences between the molecular mechanism by which CAMs and EMT signaling molecules affect the motility of cells in monolayer and MTSs. (A) Increasing confluency of monolayered cells increased the expression of CAMs and decreased cell motility through the reduction of EMT signals. The motility was further decreased by E-cadherin neutralizing antibody. (B) In MTSs, low-MW $\beta 1$ integrin enhanced EMT signals and increased cell motility. This was intensified by E-cadherin neutralizing antibody. CAM, cell adhesion molecule; EMT, epithelial-mesenchymal transition; MTS, multicellular tumor spheroid; ILK, integrin-linked kinase; Ab, antibody; MW, molecular weight.

cells metastasize to distant sites, cancer cells can change their ability to interact with the surrounding ECM and adjacent tumor cells $(11,31)$. The crosstalk between the cell-cell adhesion molecule E-cadherin and cell-ECM adhesion molecules of the integrin family contributes to the effective migration of tumor cells (13). In the present study, to mimic the early phase of metastasis, a commercial neutralizing monoclonal antibody, DECMA, that can recognize specific epitopes within the extracellular domain of E-cadherin was applied to disaggregate FRO cells from one another. The neutralizing strategy is considered to be better than a gene-silencing method because the preservation of E-cadherin synthesis is important for mesenchymal-epithelial transition, the reverse process of EMT, and the completion step of metastasis.

Although the disruption of cell-cell adhesion by blocking E-cadherin induced the downregulation of $\beta 1$ integrin in monolayer and spheroid cultures, this reduction caused differential effects on the expression of EMT signaling molecules and cell motility in the two culture conditions. These contradictory results might be elicited by the reduced MW of $\beta 1$ integrin during MTS formation because the expression levels of E-cadherin and $\beta 1$ integrin in MTSs were not markedly different from those in monolayer cells.

As a cell adhesion receptor, integrin is linked to diverse metastatic functions through intracellular signaling to other adhesion molecules or EMT molecules (32). Generally, $\beta 1$ integrin is linked to ILK, which stimulates the expression of Snail, an EMT signaling molecule. A recent study has reported that aberrant glycosylation of $\beta 1$ integrin decreases the cell-surface transport of $\beta 1$ integrin and changes the expression of ILK and Snail in hypoxic conditions (17). In fact, MTS has a spherical geometry similar to that of avascular tumor nodules in which proliferating cells are arranged at the periphery but non-proliferating cells are arranged in deeper regions under hypoxic conditions (5). Similar to these previous studies, the present study demonstrated that the expression level of ILK and Snail was enhanced by low-MW $\beta 1$ integrin, which may be regarded as aberrantly glycosylated $\beta 1$ integrin. Additionally, DECMA elicited the upregulation of ILK and Snail and accelerated the spread of MTSs.

Although the present study demonstrates that a three-dimensional culture is a more relevant model than a two-dimensional culture for studying tumor aggressiveness and metastatic potential, detailed molecular mechanisms underlying the differences in the expression of CAMs and EMT signaling molecules between these two types of cell culture environments were not elucidated. Thus, further studies are required to identify key molecular mechanisms that regulate these expression patterns during spheroid formation and confirm the in vitro results by comparing the tumorigenesis and metastasis of MTS cells with monolayer cells in vivo. Furthermore, co-culture of cancer cells with endothelial cells, fibroblasts or immune cells during spheroid formation might yield more complex models to advance metastasis research.

In conclusion, the expression levels of CAMs and the MW of $\beta 1$ integrin in MTS cells differed from those in monolayer cells, causing enhanced motility of MTS cells. The EMT signaling molecules Snail and ILK were expressed at higher levels in MTSs compared with cell monolayers. Furthermore, reduced E-cadherin binding ability increased the expression levels of EMT signaling molecules and the motility of cells. Consequently, changing monolayers to three-dimensional 
MTS not only leads to morphological changes, but also elicits marked differences in the expression of adhesion- and EMT-associated molecules.

\section{Acknowledgements}

Not applicable.

\section{Funding}

This research was supported by the Basic Science Research Program through the National Research Foundation of Korea (NRF) funded by the Ministry of Education (grant nos. NRF2 016R1D1A1A02937362 and 2018R1D1A1A09083263), Science and Technology and the Ministry of Science, ICT \& Future Planning (2017R1A2B2003575); the Korea Health Technology R\&D Project (grant nos. HI14C0748 and HI17C0387) through the Korea Health Industry Development Institute (KHIDI) by the Ministry of Health \& Welfare; the Clinical Trial Center of Korea University Anam Hospital (grant no. I1502411). This research was also supported by a grant from Korea University.

\section{Availability of data and materials}

The datasets used and/or analyzed during the current study are available from the corresponding author on reasonable request.

\section{Authors' contributions}

The authors made substantial contributions to the study as follows: BK, THK and SB contributed to study conception and design; BK, NI, TDY and JK acquired data; BK, THK and SB analyzed the data; BK, TDY, KJ, THK, SB interpreted the data. BK, THK and SB drafted the article and all other authors contributed to revising the article. All authors read and approved the final manuscript.

\section{Ethics approval and consent to participate}

Not applicable.

\section{Patient consent for publication}

Not applicable.

\section{Competing interests}

The authors declare that they have no competing interests.

\section{References}

1. Inch WR, McCredie JA and Sutherland RM: Growth of nodular carcinomas in rodents compared with multi-cell spheroids in tissue culture. Growth 34: 271-282, 1970.

2. Sutherland RM, McCredie JA and Inch WR: Growth of multicell spheroids in tissue culture as a model of nodular carcinomas. J Natl Cancer Inst 46: 113-120, 1971.

3. Breslin S and O'Driscoll L: Three-dimensional cell culture: The missing link in drug discovery. Drug Discov Today 18: 240-249, 2013.

4. Kimlin LC, Casagrande $\mathrm{G}$ and Virador VM: In vitro three-dimensional (3D) models in cancer research: An update. Mol Carcinog 52: 167-182, 2013
5. Mueller-Klieser W: Tumor biology and experimental therapeutics. Crit Rev Oncol Hematol 36: 123-139, 2000.

6. Rainaldi G, Calcabrini A, Arancia G and Santini MT: Differential expression of adhesion molecules (CD44, ICAM-1 and LFA-3) in cancer cells grown in monolayer or as multicellular spheroids. Anticancer Res 19: 1769-1778, 1999.

7. Erler JT and Weaver VM: Three-dimensional context regulation of metastasis. Clin Exp Metastasis 26: 35-49, 2009.

8. Frixen UH, Behrens J, Sachs M, Eberle G, Voss B, Warda A, Löchner D and Birchmeier W: E-cadherin-mediated cell-cell adhesion prevents invasiveness of human carcinoma cells. J Cell Biol 113: 173-185, 1991.

9. Chen A, Beetham H, Black MA, Priya R, Telford BJ, Guest J, Wiggins GA, Godwin TD, Yap AS and Guilford PJ: E-cadherin loss alters cytoskeletal organization and adhesion in non-malignant breast cells but is insufficient to induce an epithelial-mesenchymal transition. BMC Cancer 14: 552, 2014.

10. Hollestelle A, Peeters JK, Smid M, Timmermans M, Verhoog LC, Westenend PJ, Heine AA, Chan A, Sieuwerts AM, Wiemer EA, et al: Loss of E-cadherin is not a necessity for epithelial to mesenchymal transition in human breast cancer. Breast Cancer Res Treat 138: 47-57, 2013.

11. Jeanes A, Gottardi CJ and Yap AS: Cadherins and cancer: How does cadherin dysfunction promote tumor progression? Oncogene 27: 6920-6929, 2008.

12. Onder TT, Gupta PB, Mani SA, Yang J, Lander ES and Weinberg RA: Loss of E-cadherin promotes metastasis via multiple downstream transcriptional pathways. Cancer Res 68: 3645-3654, 2008

13. Canel M, Serrels A, Frame MC and Brunton VG E-cadherin-integrin crosstalk in cancer invasion and metastasis. J Cell Sci 126: 393-401, 2013.

14. Borghi N, Lowndes M, Maruthamuthu V, Gardel ML and Nelson WJ: Regulation of cell motile behavior by crosstalk between cadherin- and integrin-mediated adhesions. Proc Natl Acad Sci USA 107: 13324-13329, 2010.

15. Kaufhold S and Bonavida B: Central role of Snaill in the regulation of EMT and resistance in cancer: A target for therapeutic intervention. J Exp Clin Cancer Res 33: 62, 2014

16. Zhao D, Tang XF, Yang K, Liu JY and Ma XR: Over-expression of integrin-linked kinase correlates with aberrant expression of Snail, E-cadherin and N-cadherin in oral squamous cell carcinoma: Implications in tumor progression and metastasis. Clin Exp Metastasis 29: 957-969, 2012.

17. Takei N, Yoneda A, Sakai-Sawada K, Kosaka M, Minomi K and Tamura Y: Hypoxia-inducible ERO1 $\alpha$ promotes cancer progression through modulation of integrin- $\beta 1$ modification and signalling in HCT116 colorectal cancer cells. Sci Rep 7: 9389, 2017.

18. Livak KJ and Schmittgen TD: Analysis of relative gene expression data using real-time quantitative PCR and the 2(-Delta Delta $\mathrm{C}(\mathrm{T})$ ) method. Methods 25: 402-408, 2001.

19. De Crevoisier R, Baudin E, Bachelot A, Leboulleux S, Travagli JP, Caillou B and Schlumberger M: Combined treatment of anaplastic thyroid carcinoma with surgery, chemotherapy, and hyperfractionated accelerated external radiotherapy. Int J Radiat Oncol Biol Phys 60: 1137-1143, 2004.

20. Kebebew E, Greenspan FS, Clark OH, Woeber KA and McMillan A: Anaplastic thyroid carcinoma. Treatment outcome and prognostic factors. Cancer 103: 1330-1335, 2005.

21. Kondo T, Ezzat S and Asa SL: Pathogenetic mechanisms in thyroid follicular-cell neoplasia. Nat Rev Cancer 6: 292-306, 2006.

22. Ivanova K, Ananiev J, Aleksandrova E, Ignatova MM and Gulubova M: Expression of E-cadherin/beta-catenin in epithelial carcinomas of the thyroid gland. Open Access Maced J Med Sci 5: 155-159, 2017.

23. Brabant G, Hoang-Vu C, Cetin Y, Dralle H, Scheumann G, Mölne J, Hansson G, Jansson S, Ericson LE and Nilsson M: E-cadherin: A differentiation marker in thyroid malignancies. Cancer Res 53: 4987-4993, 1993.

24. Oliveira-Ferrer L, Legler K and Milde-Langosch K: Role of protein glycosylation in cancer metastasis. Semin Cancer Biol 44: 141-152, 2017.

25. Guo HB, Lee I, Kamar M, Akiyama SK and Pierce M: Aberrant $\mathrm{N}$-glycosylation of beta 1 integrin causes reduced alpha5beta 1 integrin clustering and stimulates cell migration. Cancer Res 62: 6837-6845, 2002.

26. Gahmberg CG, Gronholm M and Uotila LM: Regulation of integrin activity by phosphorylation. Adv Exp Med Biol 819: 85-96, 2014. 
27. Coleman DT, Soung YH, Surh YJ, Cardelli JA and Chung J: Curcumin prevents palmitoylation of integrin $\beta 4$ in breast cancer cells. PLoS One 10: e0125399, 2015.

28. Isaji T, Sato Y, Fukuda T and Gu J: N-glycosylation of the I-like domain of beta1 integrin is essential for beta1 integrin expression and biological function: Identification of the minimal N-glycosylation requirement for alpha5beta1. J Biol Chem 284: 12207-12216, 2009.

29. Marsico G, Russo L, Quondamatteo F and Pandit A: Glycosylation and integrin regulation in cancer. Trends Cancer 4: 537-552, 2018.
30. Miyoshi E, Ito Y and Miyoshi Y: Involvement of aberrant glycosylation in thyroid cancer. J Oncol 2010: 816595, 2010.

31. Wong SHM, Fang CM, Chuah LH, Leong CO and Ngai SC: E-cadherin: Its dysregulation in carcinogenesis and clinical implications. Crit Rev Oncol Hematol 121: 11-22, 2018.

32. Ganguly KK, Pal S, Moulik S and Chatterjee A: Integrins and metastasis. Cell Adh Migr 7: 251-261, 2013. 